\title{
ON STRENGTHENING OF THE KLEIMAN-CHEVALLEY PROJECTIVITY CRITERION
}

\author{
MICHAŁ FARNIK \\ (Communicated by Lev Borisov)
}

\begin{abstract}
We discuss the possibilities of strengthening the classical KleimanChevalley projectivity criterion by exploring the properties of the Picard number and the maximal quasiprojective open subsets of a variety. We also prove two theorems which give a bound on how much the criterion can be strengthened.
\end{abstract}

\section{INTRODUCTION}

The motivation for this paper comes from studying criteria for projectiveness of complete algebraic varieties. Of special interest here is the following:

Conjecture 1.1 (Chevalley). If $X$ is a normal complete algebraic variety such that for every finite set $S \subset X$ there is an affine open subset $U \subset X$ such that $S \subset U$, then $X$ is projective.

This conjecture has been proven for $\mathbb{Q}$-factorial varieties by Kleiman in [7] and by Włodarczyk in [12. However it fails to be true if we omit the assumption of $X$ being normal, as shown in [2]. Our goal is to set exact boundaries of how much one can weaken or must strengthen the assumptions of Chevalley conjecture in order for it to be true. The following three values will be crucial in our considerations:

$\rho(X)$ - the Picard number of $X$, i.e. the rank of $\operatorname{Pic}(X)$ or the Néron-Severi group.

$m q o s(X)$ - the cardinality of $M Q O S(X)$, the set of maximal quasiprojective open subsets of $X$.

$a(X):=\sup \{n$ : every set of $n$ points in $X$ is contained in some open affine subset of $X\}$.

Obviously for projective varieties we have $\rho(X) \geq 1, \operatorname{mqos}(X)=1, a(x)=\infty$; thus the truly interesting thing is what combinations of these three values are (im)possible for nonprojective varieties.

Kleiman's result can in fact be stated as follows:

Theorem 1.2 (Kleiman, [7, Chapter IV, $\S 2$, Corollary 2]). If $X$ is a $\mathbb{Q}$-factorial complete algebraic variety and $a(X) \geq 2 \rho(X)$, then $X$ is projective.

Received by the editors June 15, 2011 and, in revised form, January 7, 2012.

2010 Mathematics Subject Classification. Primary 51N35; Secondary 14A10, 14M25.

Key words and phrases. Picard number, maximal open quasiprojective set, Chevalley projectivity criterion, nonprojective varieties, toric varieties.

The author was partially supported by Polish MNiSW grant N N201 611740, 2011-2012. 
In [8, VI.2.20] Kollár introduced a simple modification in Kleiman's proof to obtain that it is enough to assume $a(X) \geq \rho(X)+1$. Theorem 2.2 yields that the weakest assumption with which Theorem 1.2 may be true is $a(X) \geq \rho(X)-1$.

Włodarczyk uses the simple fact that $a(X) \geq m q o s(X)$ implies projectivity. His main result is that a large class of varieties (including $\mathbb{Q}$-factorial ones) always has finite $\operatorname{mqos}(X)$. There is a natural question of how $\rho(X)$ and $m q o s(X)$ are related, especially whether $\operatorname{mqos}(X)$ can be bounded by some multiple of $\rho(X)$. Theorem 2.1 says that there exist smooth varieties with $m q o s(X)=(\rho(X)-1)$ !, and Theorem 3.1 gives the existence of normal varieties with $\rho(X)=0$ and arbitrary large $a(X)$, and thus also arbitrary large $\operatorname{mqos}(X)$.

The existence of varieties with $\rho(X)=0$ is a major obstacle while trying to adapt various arguments from the $\mathbb{Q}$-factorial case to normal varieties. In section 4 we comment on why a theorem crucial in [7] fails to be true for normal varieties.

\section{The SMooth EXAmples}

In this section we prove the following:

Theorem 2.1. For every integer $n \geq 2$ there exists a smooth complete variety $X$ with $\rho(X)=n+1$ and $\operatorname{mqos}(X)=n$ !.

Theorem 2.2. For every integer $n \geq 2$ there exists a smooth complete variety $X^{\prime}$ with $\rho\left(X^{\prime}\right)=n+1$ and $a\left(X^{\prime}\right)=n-1$.

First we construct a variety $X$ satisfying the conditions of Theorem 2.1. Then we show how to modify the construction to obtain a variety $X^{\prime}$ satisfying the conditions of Theorem 2.2. Finally we comment on what other kinds of results can be obtained using the presented method.

The construction is over an algebraically closed field. It is a generalization of the well known construction of a nonprojective threefold due to Hironaka (see 4] or [10, Chapter VI, 2.3]). We obtain Hironaka's example by taking $n=2$.

Proof of Theorem 2.1. Let $C_{1}, \ldots, C_{n}$ be smooth curves in $\mathbb{P}^{3}$ such that each one intersects every other transversally and at least in two points, and the intersection of any three is empty. For simplicity we can take cubics on a plane. Let $S=\left\{P_{1}, \ldots, P_{k}\right\}$ be the set of all intersection points of the curves $C_{i}$. Let $\pi_{S}: X_{S} \rightarrow \mathbb{P}^{3} \backslash S$ be the blowup along $\bigcup C_{i} \backslash S$.

For every point $P_{t}$ being the intersection of $C_{i}$ with $C_{j}$, let $\pi_{i, j}: X_{t}^{i, j} \rightarrow \mathbb{P}^{3} \backslash$ $\left(S \backslash P_{t}\right)$ be the blowup first along $C_{i} \backslash\left(S \backslash P_{t}\right)$, then along the proper transform of $C_{j} \backslash\left(S \backslash P_{t}\right)$, and finally along $\bigcup_{k \neq i, j} C_{k} \backslash S$ on which the previous blowups are isomorphisms.

Note that $\pi_{i, j}^{-1}\left(\mathbb{P}^{3} \backslash S\right) \cong X_{S}$ and $\pi_{i, j}^{-1}\left(P_{t}\right)$ are two copies of $\mathbb{P}^{1}$ meeting in a point. Indeed, if $\pi_{1}$ is the blowup along $C_{i} \backslash\left(S \backslash P_{t}\right)$, then $E^{\prime}=\pi_{1}^{-1}\left(P_{t}\right)$ is a line meeting $C^{\prime}$, the proper transform of $C_{j} \backslash\left(S \backslash P_{t}\right)$, transversally in a point $A$. Further, if $\pi_{2}$ is the blowup along $C^{\prime}$, then $F=\pi_{2}^{-1}(A)$ is also a line. Obviously it meets $E$, the proper transform of $E^{\prime}$. The final blowup of other curves is an isomorphism on $E \cup F$. The two lines being $\pi_{i, j}^{-1}\left(P_{t}\right)$ and originating from the first and second blowup will be denoted respectively $E_{t}^{i, j}$ and $F_{t}^{i, j}$.

Elementary calculus shows that the natural isomorphism $X_{t}^{i, j} \backslash\left(E_{t}^{i, j} \cup F_{t}^{i, j}\right) \rightarrow$ $X_{S} \rightarrow X_{t}^{j, i} \backslash\left(E_{t}^{j, i} \cup F_{t}^{j, i}\right)$ extends to an isomorphism $X_{t}^{i, j} \backslash E_{t}^{i, j} \rightarrow X_{t}^{j, i} \backslash E_{t}^{j, i}$; however it does not extend to $X_{t}^{i, j} \rightarrow X_{t}^{j, i}$. 
For every $t$ we choose one of the two varieties $X_{t}^{i, j}$ and $X_{t}^{j, i}$ and call it $X_{t}$. We make the choice in such a way that for every $C_{i}$ and $C_{j}$ there is at least one $X_{t_{1}}=X_{t_{1}}^{i, j}$ and at least one $X_{t_{2}}=X_{t_{2}}^{j, i}$. We construct the variety $X$ by glueing all the $X_{t}$ along their common open subset $X_{S}$.

Obviously $\rho(X)=n+1$, the other desired properties of $X$, follow from Lemma 2.3 and Theorem 2.6 .

Lemma 2.3. The variety $X$ constructed in Theorem 2.1 is nonsingular and complete.

Proof. The variety $X$ is nonsingular because it is covered by open subsets $X_{t}$ which are blowups of nonsingular varieties along nonsingular subsets, thus nonsingular.

Obviously $\mathbb{P}^{3}$ is complete; hence to prove that $X$ is complete it suffices to show that the projection $\pi: X \rightarrow \mathbb{P}^{3}$ is closed. Note that $\pi: X_{t} \rightarrow \mathbb{P}^{3} \backslash\left(S \backslash P_{t}\right)$ is a composition of blowups which are closed. The sets $\mathbb{P}^{3} \backslash\left(S \backslash P_{t}\right)$ are open subsets covering $\mathbb{P}^{3}$ and being closed is a local property; thus $X$ is complete.

Lemma 2.4. Let $X$ be a complete nonsingular variety and let $C_{1}, \ldots, C_{n}$ be effective curves in $X$ whose sum is numerically equivalent to 0 . Then any open subset $U \subset X$ such that $U \cap C_{i} \neq \emptyset$ for every $i$ cannot be quasiprojective.

Proof. Suppose such $U$ is quasiprojective. Then we have $U \subset \mathbb{P}^{k}$ for some $k$. Take a hyperplane section $H$ such that $H \cap U \cap C_{i}$ is nonempty and finite for all $i$. Let $D=H \cap U$; then the closure of $D$ in $X$ is a divisor with positive intersection with every $C_{i}$, a contradiction with $\sum C_{i} \equiv 0$.

Lemma 2.5. For every cycle $i_{0}, i_{1}, \ldots, i_{k}=i_{0}$ of indices and $t_{0}, t_{1}, \ldots, t_{k}=t_{0}$ such that $P_{t_{j}} \in C_{i_{j-1}} \cap C_{i_{j}}$ and $X_{t_{j}}=X_{t_{j}}^{i_{j-1}, i_{j}}$, the sum $\sum_{j=1}^{k} E_{t_{j}}^{i_{j-1}, i_{j}}$ is linearly equivalent to 0 in $X$.

Proof. Let $E_{i_{j}}$ be the fibre of $\pi_{S}$ at some point of $C_{i_{j}} \backslash S$. Then $E_{i_{j}}$ is linearly equivalent to both $E_{t_{j+1}}^{i_{j}, i_{j+1}}+F_{t_{j+1}}^{i_{j}, i_{j+1}}$ and $F_{t_{j}}^{i_{j-1}, i_{j}}$. Adding up, we see that $\sum_{j=1}^{k} E_{i_{j}}$ is equivalent to $\sum_{j=1}^{k} E_{t_{j}}^{i_{j-1}, i_{j}}+\sum_{j=1}^{k} F_{t_{j}}^{i_{j-1}, i_{j}}$ when going to where a curve was the first to be blown up and equivalent to $\sum_{j=1}^{k} F_{t_{j}}^{i_{j-1}, i_{j}}$ when going the other way. The assertion follows from those two equivalences.

Let $\sigma \in S_{n}$ be a permutation. Let $X_{\sigma}$ be the variety obtained from $X$ by cutting out all the lines $E_{t}^{\sigma(j), \sigma(i)}$ for all $i<j$ and relevant $t$. Then we have the following:

Theorem 2.6. The variety $X$ from Theorem 2.1 has exactly $n$ ! maximal open quasiprojective subsets, namely the varieties $X_{\sigma}$ for all $\sigma \in S_{n}$.

Proof. First we will show that every $X_{\sigma}$ is quasiprojective. Indeed, it is obtained by glueing together some of the varieties $X_{t}^{\sigma(i), \sigma(j)}$ and some of $X_{t}^{\sigma(j), \sigma(i)} \backslash E_{t}^{\sigma(j), \sigma(i)}$ for all $i<j$. The latter varieties are isomorphic to $X_{t}^{\sigma(i), \sigma(j)} \backslash E_{t}^{\sigma(i), \sigma(j)}$. This shows that $X_{\sigma}$ is an open subset of the variety obtained from $\mathbb{P}^{3}$ by consecutive blowing up of the proper transforms of the curves $C_{\sigma(1)}, \ldots, C_{\sigma(n)}$, thus quasiprojective.

To show that $X_{\sigma}$ are the only maximal open quasiprojective subsets of $X$, take an open quasiprojective subset $U \subset X$. From Lemmas 2.4 and 2.5 we know that $U$ has to be cycle free. Thus there exists an index $i_{1}$ such that all the curves $E_{t}^{j, i_{1}}$ for $j \neq i_{1}$ have been cut out; otherwise we would be able to construct a cycle. We 
set $i_{k}$ to be an index such that all the curves $E_{t}^{j, i_{k}}$ for $j \notin\left\{i_{1}, \ldots, i_{k-1}\right\}$ have been cut out. We obtain a permutation $\sigma(k)=i_{k}$ such that $U$ is a subset of $X_{\sigma}$.

We proceed with construction of $X^{\prime}$. It is very similar to the construction of $X$; thus we only highlight the differences.

Proof of Theorem 2.2. Let $C_{1}^{\prime}, \ldots, C_{n}^{\prime}=C_{0}^{\prime}$ be smooth curves in $\mathbb{P}^{3}$ such that each $C_{i}^{\prime}$ intersects $C_{i-1}^{\prime}$ and $C_{i+1}^{\prime}$ transversally in different points and does not intersect the other curves. For simplicity we can take suitable lines. Let $P_{i}$ be the intersection point of $C_{i}^{\prime}$ and $C_{i-1}^{\prime}$. The variety $X^{\prime}$ is obtained by blowing up $\mathbb{P}^{3}$ along $C_{1}^{\prime}, \ldots, C_{n}^{\prime}$ in such way that in a neighbourhood of $P_{i}$ the curve $C_{i}^{\prime}$ is blown up after $C_{i-1}^{\prime}$. Note that in $X^{\prime}$ there is exactly one cycle as described in Lemma 2.5. namely $1,2, \ldots, n, 1$. Analogously as with $X$, we obtain that the maximal open quasiprojective subsets of $X^{\prime}$ are of the form $X^{\prime} \backslash E_{i}$, where $E_{i}$ is the exceptional divisor of the first blowup in $P_{i}$. Thus every set not contained in an affine subset of $X^{\prime}$ contains a point from each $E_{i}$. This gives that $a\left(X^{\prime}\right)=n-1$.

One can obtain various other results using this method. It is easy to describe the possible constructions in terms of graph theory. We assign a directed multigraph $G=(V, \vec{E})$ to a variety $X$ as follows: the vertices $v_{i}$ correspond to the curves $C_{i}$ blown up, and the edges $v_{i} v_{j}$ correspond to points of $X$ where $C_{i}$ was blown up before $C_{j}$. In the constructions above the assigned graphs where respectively a complete graph and a cycle. The acyclic subgraphs of $G$ correspond to some of the quasiprojective open subsets of $X$; the maximal, with respect to inclusion, acyclic subgraphs correspond to elements of $M Q O S(X)$. The value $a(X)$ is equal to the length of the shortest cycle minus one.

It is worth mentioning that a variety $X_{t}^{j, i}$ can be obtained from $X_{t}^{i, j}$ by flopping the line $E_{t}^{i, j}$ (see [5] for details). This flop is an isomorphism outside line $E_{t}^{i, j}$ and may be carried out on any variety containing $X_{t}^{i, j}$ as an open subset. In terms of the corresponding graphs such a flop would mean reversing the direction of the corresponding edge. This leads to an alternative construction of the varieties $X$ and $X^{\prime}$. One can blow up along the curves in any order and then flop appropriate lines to obtain the desired result.

\section{THE TORIC EXAMPLES}

In this section we prove the following theorem:

Theorem 3.1. For every $t \geq 5$ there exists a complete normal toric variety $X$ with $a(X)=t$ and $\rho(X)=0$.

We use a characterization of the conditions $a(X)=n$ and $\rho(X)=0$ in terms of continuous piecewise linear functions on $|\Delta|$, the support of the fan associated with the toric variety $X$.

We assume the reader has some background knowledge on toric varieties. For more details see e.g. [3, sections 3.3, 3.4].

In this section a divisor means a Cartier divisor equivariant under the torus action. We can restrict ourselves to such divisors when studying $a(X)$ and $\rho(X)$. There is a one-to-one correspondence between the divisors $D$ on $X$ and continuous 
functions $\Psi_{D}$ on $|\Delta|$ given on each cone by an element of the dual lattice (thus integral and linear on each cone). Integral linear functions are defined by the same element of the dual lattice (for cones of maximal dimension) and thus correspond to principal divisors. This yields the following:

Remark 3.2. For a toric variety $X$ given by a fan $\Delta$, we have: $\operatorname{Pic}(X)=0$ iff every continuous integral piecewise linear function on the support $|\Delta|$ is linear.

Let $\psi$ be a piecewise linear function on the support of a fan $\Delta$. We call $\psi$ strictly convex if for every $\sigma \in \Delta$ there exists a linear function $L$ such that $\psi=L$ on $\sigma$ and $\psi>L$ elsewhere. We denote such a linear function by $\psi_{\sigma}$. If the cone $\sigma$ is of maximal dimension, then the linear function is uniquely determined. Otherwise we use the notation $\psi_{\sigma}$ only after specifying which of the candidates we choose. Note also that while examining convexity, we may restrict ourselves to the maximal cones.

To characterize the condition $a(X)=n$ we use the following theorem:

Theorem 3.3. A divisor $D$ on a toric variety is ample iff the corresponding function $\Psi_{D}$ is strictly convex.

Proof. See [9, page 48] or [3, section 4] for complete varieties.

Now we can state the following:

Theorem 3.4. For a toric variety $X$ given by a fan $\Delta$ we have: $a(X) \geq n$ iff for any $\sigma_{1}, \ldots, \sigma_{n}$ in $\Delta$ there exists a continuous piecewise linear function on $\bigcup_{i=1}^{n} \sigma_{i}$ which is strictly convex. Furthermore $a(X) \leq n$ iff there are $\sigma_{1}^{\prime}, \ldots, \sigma_{n}^{\prime}, \sigma_{n+1}^{\prime}$ in $\Delta$ such that there is no strictly convex continuous piecewise linear function on $\bigcup_{i=1}^{n+1} \sigma_{i}^{\prime}$.

Proof. The proof will be similar to the one of Proposition 5.1 in [12]. We will prove the first statement; the second follows by contraposition.

Assume $a(X) \geq n$ and fix $\sigma_{1}, \ldots, \sigma_{n}$ in $\Delta$. Let $P_{1}, \ldots, P_{n}$ be points in orbits corresponding to $\sigma_{1}, \ldots, \sigma_{n}$. Let $X^{\prime}$ be the image under the torus action of an open affine subset $U$ of $X$ containing $P_{1}, \ldots, P_{n}$. By [9, Chapter I, §2] the image of an open quasiprojective subset under the torus action is again open and quasiprojective. Thus $X^{\prime}$ is an open quasiprojective toric subvariety of $X$. The fan $\Delta^{\prime}$ of $X^{\prime}$ consists of those cones in $\Delta$ whose corresponding orbits intersect $U$, in particular, $\sigma_{1}, \ldots, \sigma_{n}$. Any of the ample divisors on $X^{\prime}$ will yield the required function.

Now let $P_{1}, \ldots, P_{n}$ be points in $X$. Let $\sigma_{1}, \ldots, \sigma_{n}$ be cones corresponding to orbits containing $P_{1}, \ldots, P_{n}$. Let $\Delta^{\prime}$ be the fan consisting of $\sigma_{1}, \ldots, \sigma_{n}$ and their faces, and let $X^{\prime}$ be the corresponding toric open subvariety of $X$. The strictly convex continuous piecewise linear function on $\left|\Delta^{\prime}\right|$ gives an ample divisor on $X^{\prime}$. Since $X^{\prime}$ contains $P_{1}, \ldots, P_{n}$ and is quasiprojective, we can easily find an open affine subset containing $P_{1}, \ldots, P_{n}$.

Proof of Theorem 3.1. We start by constructing a fan $\Delta^{\prime}$ of a projective toric variety $X^{\prime}$ with $\rho\left(X^{\prime}\right)=1$. Afterwards we show how to modify $\Delta^{\prime}$ to obtain a fan $\Delta$ of a toric variety with $\rho(X)=0$. For simplicity we do not distinguish between cones in $\mathbb{R}^{3}$ and $\mathbb{Z}^{3}$; we construct cones in $\mathbb{R}^{3}$ and assume an intersection with $\mathbb{Z}^{3}$ is taken when required. We also assume that $t=2 n+2$, where $n$ is even; however in Remark 3.5 we comment on how to drop this assumption. 
Let $T$ be the convex polytope whose vertices are the points $P_{1}, \ldots, P_{4 n}$, $Q_{1}, \ldots, Q_{4 n}, Q_{0}$, where for $k=1, \ldots, \frac{n}{2}$ we take:

$P_{k}=\left(n k+k^{2}, n^{2}-k^{2},-n^{2}\right)$,

$P_{\frac{n}{2}+k}=\left(n^{2}-\left(\frac{n}{2}-k\right)^{2}, n\left(\frac{n}{2}-k\right)+\left(\frac{n}{2}-k\right)^{2},-n^{2}\right)$,

$P_{n+k}=\left(n^{2}-k^{2},-n k-k^{2},-n^{2}\right)$,

$P_{\frac{3 n}{2}+k}=\left(n\left(\frac{n}{2}-k\right)+\left(\frac{n}{2}-k\right)^{2},-n^{2}+\left(\frac{n}{2}-k\right)^{2},-n^{2}\right)$,

$P_{2 n+k}=\left(-n k-k^{2},-n^{2}+k^{2},-n^{2}\right)$,

$P_{\frac{5 n}{2}+k}=\left(-n^{2}+\left(\frac{n}{2}-k\right)^{2},-n\left(\frac{n}{2}-k\right)-\left(\frac{n}{2}-k\right)^{2},-n^{2}\right)$,

$P_{3 n+k}=\left(-n^{2}+k^{2}, n k+k^{2},-n^{2}\right)$,

$P_{\frac{7 n}{2}+k}=\left(-n\left(\frac{n}{2}-k\right)-\left(\frac{n}{2}-k\right)^{2}, n^{2}-\left(\frac{n}{2}-k\right)^{2},-n^{2}\right)$.

Thus the points $P_{1}, \ldots, P_{4 n}$ span a $4 n$-gon in the $z=-n^{2}$ plane and are indexed clockwise.

Further:

$Q_{k}=P_{k}+\left(0,0,2 n^{2}\right)$ for $k=1, \ldots, 2 n-1,2 n+1, \ldots, 4 n-1$,

$Q_{2 n}=\left(0,-n^{2}, n^{2}-\frac{n^{2}}{n^{2}-1}\right), Q_{4 n}=\left(0, n^{2}, n^{2}-\frac{n^{2}}{n^{2}-1}\right)$,

$Q_{0}=\left(0,0,2 n^{2}\right)$.

Now let $\Delta^{\prime}$ be the set of cones spanned over $\mathbb{R}_{+}$by the faces, edges and vertices of $T$ and $(0,0,0)$. Let $\sigma\left(P_{i_{1}}, \ldots, P_{i_{k}}\right)$ denote the cone spanned over $\mathbb{R}_{+}$by the set of points $\left\{P_{i_{1}}, \ldots, P_{i_{k}}\right\}$. The fan $\Delta^{\prime}$ has the following $8 n-1$ maximal cones:

$\sigma_{0}=\sigma\left(P_{1}, \ldots, P_{4 n}\right), \sigma_{1}=\sigma\left(P_{4 n}, P_{1}, Q_{4 n}, Q_{1}\right)$,

$\sigma_{k}=\sigma\left(P_{k-1}, P_{k}, Q_{k-1}, Q_{k}\right)$ for $k=2, \ldots, 4 n$,

$\sigma_{4 n+1}=\sigma\left(Q_{0}, Q_{4 n-1}, Q_{4 n}, Q_{1}\right), \sigma_{6 n+1}=\sigma\left(Q_{0}, Q_{2 n-1}, Q_{2 n}, Q_{2 n+1}\right)$,

$\sigma_{4 n+k}=\sigma\left(Q_{0}, Q_{k-1}, Q_{k}\right)$ for $k=2, \ldots, 2 n-1,2 n+2, \ldots, 2 n-1$.

To show that $X^{\prime}$ is projective (and thus $a\left(X^{\prime}\right)=\infty$ ), let $\phi$ be the unique piecewise linear function on $\Delta^{\prime}$ whose value is 1 on every vertex of $T$. It is strictly convex because $T$ is convex and does not have two coplanar faces. It is not integral; however some multiple of it is integral and thus corresponds to an ample divisor.

To see that $\rho\left(X^{\prime}\right)=1$ it is enough to observe that any piecewise linear function $\phi^{\prime}$ on $\Delta^{\prime}$ is a combination of the function $\phi$ and a linear function. Indeed fix a function $\phi^{\prime}$; by adding a suitable linear function, we may assume that it has value 0 on one of the cones. Let $\phi^{\prime}$ have value 0 on $\sigma_{0}$ and $\phi^{\prime}\left(Q_{1}\right)=a$. Let $\phi^{\prime \prime}$ be obtained by subtracting from $\phi^{\prime}$ the function $\frac{a}{2} \phi$ and the linear function $(x, y, z) \mapsto \frac{a z}{n^{2}}$. Obviously $\phi^{\prime \prime}$ has value 0 on $\sigma_{0}$ and on $Q_{1}$. Any three rays of a cone span $\mathbb{R}^{3}$ over $\mathbb{R}$; thus if a piecewise linear function has value 0 along three rays of a cone, then it has value 0 on the whole cone. Since $\phi^{\prime \prime}\left(P_{1}\right)=\phi^{\prime \prime}\left(P_{2}\right)=\phi^{\prime \prime}\left(Q_{1}\right)=0$, we have that $\left.\phi^{\prime \prime}\right|_{\sigma_{2}}=0$ and $\phi^{\prime \prime}\left(Q_{2}\right)=0$. Using this argument on $\sigma_{3}, \ldots, \sigma_{4 n}$ and $\sigma_{4 n+1}$, we obtain that $\phi^{\prime \prime} \equiv 0$ and thus $\phi^{\prime}$ is a combination of $\phi$ and a linear function.

We proceed with the construction of $X$. Let $\Delta$ be the fan obtained from $\Delta^{\prime}$ by modifying all cones containing the ray through $P_{4 n}$ to contain the ray through $P_{0}=\left(0, n^{2},-n^{2}-\varepsilon\right)$ instead, where $\varepsilon$ is small, positive and rational. The affected maximal cones will be $\sigma_{0}, \sigma_{1}$ and $\sigma_{4 n}$. The fan $\Delta$ can no longer be obtained from a polytope. Let $X$ be the toric variety associated with $\Delta$. The condition $a(X)=2 n+2$ is satisfied for $\varepsilon \leq \frac{1}{2 n^{2}}$ when $n \geq 4$ and for $\varepsilon \leq \frac{1}{23}$ when $n=2$.

To prove that $\rho(X)=0$ we show that every piecewise linear function $\phi$ on $\Delta$ is linear. Again we may assume that $\left.\phi\right|_{\sigma_{0}}=0$. Let $\phi\left(Q_{1}\right)=a$. From linearity of $\phi$ on $\sigma_{1}$ we obtain that $\left(\phi\left(Q_{1}\right)-\phi\left(P_{1}\right)\right)\left(2 n^{2}-\frac{n^{2}}{n^{2}-1}+\varepsilon\right)=\left(\phi\left(Q_{4 n}\right)-\phi\left(P_{4 n}\right)\right) 2 n^{2}$ 
and thus $\phi\left(Q_{4 n}\right)=a\left(1-\frac{1}{2 n^{2}-2}+\frac{\varepsilon}{2 n^{2}}\right)$. From linearity of $\phi$ on $\sigma_{4 n}$ we obtain $\phi\left(Q_{4 n-1}\right)=a$, and from linearity on $\sigma_{2}, \ldots, \sigma_{2 n-1}$ and $\sigma_{4 n-1}, \ldots, \sigma_{2 n+2}$ we obtain $\phi\left(Q_{2}\right)=\ldots=\phi\left(Q_{2 n-1}\right)=a$ and $\phi\left(Q_{4 n-2}\right)=\ldots=\phi\left(Q_{2 n+1}\right)=a$. From linearity on $\sigma_{2 n}$ we obtain $\phi\left(Q_{2 n}\right)=a\left(1-\frac{1}{2 n^{2}-2}\right)$. Finally, calculating $\phi\left(Q_{0}\right)$ using the linearity on $\sigma_{4 n+1}$ and $\sigma_{6 n+1}$, we obtain $\phi\left(Q_{0}\right)=\frac{3 a}{2}+a \varepsilon \frac{n^{2}-1}{2 n^{2}}=\frac{3 a}{2}$. Since $\varepsilon \neq 0$ this equality can hold only if $a=0$ and thus $\phi \equiv 0$.

Note that in fact we have shown that any piecewise linear function defined on $\sigma_{0}, \sigma_{4 n+1}, \sigma_{6 n+1}$ and $\sigma_{1}, \ldots, \sigma_{2 n}$ has to be linear on the sum of those cones and thus cannot give rise to an ample divisor. This yields that $a(X) \leq 2 n+2$.

Now we show that $a(X) \geq 2 n+2$. To do so, we show that it is enough to exclude either $\sigma_{4 n+1}$ or $\sigma_{6 n+1}$ from $\Delta$ to make the associated variety quasiprojective. Then we show that excluding from $\Delta$ cones $\sigma_{i} \in\left\{\sigma_{2}, \ldots, \sigma_{2 n-1}\right\}$ and $\sigma_{j} \in\left\{\sigma_{2 n+2}, \ldots, \sigma_{4 n-1}\right\}$ enables us to "combine" the strictly convex piecewise linear functions on $\left|\Delta \backslash\left\{\sigma_{4 n+1}\right\}\right|$ and $\left|\Delta \backslash\left\{\sigma_{6 n+1}\right\}\right|$ into a strictly convex piecewise linear function on $\left|\Delta \backslash\left\{\sigma_{i}, \sigma_{j}\right\}\right|$. Finally, we show that excluding $\sigma_{0}$ or both $\sigma_{4 n}$ and $\sigma_{1}$ or both $\sigma_{2 n}$ and $\sigma_{2 n+1}$ also gives rise to a quasiprojective variety. This leads to the conclusion that a subfan of $\Delta$ defines a variety that is quasiprojective unless it contains $\sigma_{0}, \sigma_{4 n+1}, \sigma_{6 n+1}, \sigma_{4 n}$ or $\sigma_{1}, \sigma_{2 n}$ or $\sigma_{2 n+1}$ and either $\sigma_{2}, \ldots, \sigma_{2 n-1}$ or $\sigma_{2 n+2}, \ldots, \sigma_{4 n-1}$. So a finite subset of $X$ is contained in some quasiprojective open subset of $X$ unless it contains an appropriate choice of points corresponding to the cones stated above. Thus every set of at most $2 n+2$ points from $X$ is contained in some quasiprojective open subset of $X$ and thus $a(X) \geq 2 n+2$.

We proceed with defining convex piecewise linear functions on the required subfans of $\Delta$. In every case it is enough to define the function on $P_{1}, \ldots, P_{4 n}$, $Q_{0}, \ldots, Q_{4 n}$ as it uniquely determines a piecewise linear function.

For $\left|\Delta \backslash\left\{\sigma_{4 n+1}\right\}\right|$ we take $\phi\left(P_{1}\right)=\ldots=\phi\left(P_{4 n-1}\right)=\phi\left(Q_{0}\right)=\ldots=\phi\left(Q_{4 n-1}\right)=1$ and $\phi\left(P_{4 n}\right)=\phi\left(Q_{4 n}\right)=1+\frac{\varepsilon}{n^{2}}$. The convexity is obvious.

For $\left|\Delta \backslash\left\{\sigma_{6 n+1}\right\}\right|$ we take $\phi\left(P_{1}\right)=\ldots=\phi\left(P_{4 n-1}\right)=\phi\left(Q_{0}\right)=1, \phi\left(P_{4 n}\right)=1+\frac{\varepsilon}{n^{2}}$, $\phi\left(Q_{1}\right)=\ldots=\phi\left(Q_{4 n-1}\right)=a$ and $\phi\left(Q_{4 n}\right)=b$. The values $a$ and $b$ are already fixed by the condition of piecewise linearity on $\sigma_{1}, \sigma_{4 n}$ and $\sigma_{4 n+1}$. Elementary calculations reveal $a=1+\frac{2 \varepsilon\left(n^{2}-1\right)}{3 n^{2}-\varepsilon\left(n^{2}-1\right)}$ and $b=1+\frac{2 \varepsilon}{3 n^{2}+\varepsilon\left(n^{2}-1\right)}$. Again, the convexity is quite obvious.

For $\left|\Delta \backslash\left\{\sigma_{i}, \sigma_{j}\right\}\right|$, where $i \in\{2, \ldots, 2 n-1\}$ and $j \in\{2 n+2, \ldots, 4 n-1\}$, we take $\phi\left(P_{1}\right)=\ldots=\phi\left(P_{4 n-1}\right)=\phi\left(Q_{0}\right)=1, \phi\left(P_{4 n}\right)=1+\frac{\varepsilon}{n^{2}}, \phi\left(Q_{4 n}\right)=b$, $\phi\left(Q_{1}\right)=\ldots=\phi\left(Q_{i-1}\right)=\phi\left(Q_{j}\right)=\ldots=\phi\left(Q_{4 n-1}\right)=a$ and $\phi\left(Q_{i}\right)=\ldots=$ $\phi\left(Q_{j-1}\right)=1$, where $a$ and $b$ are as above. Note that on every cone except for $\sigma_{4 n+i}$ and $\sigma_{4 n+j}$ this function is equal to one of the functions defined for $\left|\Delta \backslash\left\{\sigma_{4 n+1}\right\}\right|$ and $\left|\Delta \backslash\left\{\sigma_{6 n+1}\right\}\right|$. Obviously the function is convex separately on each of those parts, and we only need to verify inequalities involving both parts. In fact what we need to verify is that $\phi_{\sigma_{i-1}}\left(Q_{i}\right)<\phi\left(Q_{i}\right), \phi_{\sigma_{i+1}}\left(Q_{i-1}\right)<\phi\left(Q_{i-1}\right), \phi_{\sigma_{4 n+i-1}}\left(Q_{i}\right)<\phi\left(Q_{i}\right)$, $\phi_{\sigma_{4 n+i}}\left(Q_{i-2}\right)<\phi\left(Q_{i-2}\right), \phi_{\sigma_{4 n+i}}\left(Q_{i+1}\right)<\phi\left(Q_{i+1}\right), \phi_{\sigma_{4 n+i+1}}\left(Q_{i-1}\right)<\phi\left(Q_{i-1}\right)$ and the same inequalities with $j$ instead of $i$. If these inequalities hold, then so will the required inequalities for other cones and points $P$ and $Q$, and from inequalities on points $P$ and $Q$ follow the inequalities on whole cones. The calculations are elementary yet tiresome, and we will omit them. The important fact is that they hold if $\varepsilon \leq \frac{1}{2 n^{2}}$ for $n \geq 4$ and $\varepsilon \leq \frac{1}{23}$ for $n=2$. Note that the $\varepsilon$ chosen must be rational in order for $\phi$ to have an integral multiple. 
For $\left|\Delta \backslash\left\{\sigma_{0}\right\}\right|$ we take $\phi\left(P_{1}\right)=\ldots=\phi\left(P_{4 n}\right)=\phi\left(Q_{0}\right)=\ldots=\phi\left(Q_{4 n}\right)=1$. The convexity is obvious.

For $\left|\Delta \backslash\left\{\sigma_{4 n}, \sigma_{1}\right\}\right|$ we take $\phi\left(P_{1}\right)=\ldots=\phi\left(P_{4 n-1}\right)=\phi\left(Q_{0}\right)=\ldots=\phi\left(Q_{4 n}\right)=1$ and $\phi\left(P_{4 n}\right)=1+\frac{\varepsilon}{n^{2}}$. The convexity is obvious except for the cone $\sigma\left(P_{4 n}, Q_{4 n}\right)$, which is now maximal but not of maximal dimension. The values on $P_{4 n}$ and $Q_{4 n}$ and linearity require the linear function defining $\phi$ on $\sigma\left(P_{4 n}, Q_{4 n}\right)$ to be of the form $(x, y, z) \mapsto \alpha x+(1+\varepsilon)\left(2-\frac{1}{n^{2}-1}\right) \frac{y}{\beta}-\frac{\varepsilon z}{n^{2} \beta}$, where $\alpha \in \mathbb{R}$ and $\beta=2 n^{2}+\varepsilon-\frac{n^{2}}{n^{2}-1}$. For the most intuitive choice $\alpha=0$ it is easy to verify the convexity.

Finally, for $\left|\Delta \backslash\left\{\sigma_{2 n}, \sigma_{2 n+1}\right\}\right|$ we take $\phi\left(P_{1}\right)=\ldots=\phi\left(P_{4 n-1}\right)=\phi\left(Q_{0}\right)=1$, $\phi\left(P_{4 n}\right)=1+\frac{\varepsilon}{n^{2}}, \phi\left(Q_{1}\right)=\ldots=\phi\left(Q_{2 n-1}\right)=\phi\left(Q_{2 n+1}\right)=\ldots=\phi\left(Q_{4 n-1}\right)=a$ and $\phi\left(Q_{2 n}\right)=\phi\left(Q_{4 n}\right)=b$ with $a$ and $b$ as above. The cone $\sigma\left(P_{4 n}, Q_{4 n}\right)$ is maximal but not of maximal dimension and again there is a family of linear functions that coincide with $\phi$ on $\sigma\left(P_{4 n}, Q_{4 n}\right)$. These functions are $(x, y, z) \mapsto \alpha x-\left(\frac{1}{n^{2}}+\beta\right) y+\beta z$, where $\alpha \in \mathbb{R}$ and $\beta=\frac{2\left(n^{2}-1\right) \varepsilon}{n^{2}\left(2 n^{2}-3\right)\left(3 n^{2}+\varepsilon\left(n^{2}-1\right)\right)}$. For $\alpha=0$ the convexity is easy to verify.

Remark 3.5. The assumption $t=2 n+2$ where $n$ is even is not essential.

Proof. Notice that if we define $T$ to be the convex polytope on vertices $P_{1}, \ldots, P_{n-1}$, $P_{n+1}, \ldots, P_{4 n}, Q_{1}, \ldots, Q_{n-1}, Q_{n+1}, \ldots, Q_{4 n}, Q_{0}$, then the construction used in the proof of Theorem 3.1 gives a variety $X$ with $a(X)=2 n+1$. This is because now we have a cone $\sigma_{n}^{\prime}=\sigma\left(P_{n-1}, P_{n+1}, Q_{k-1}, Q_{n+1}\right)$ instead of cones $\sigma_{n}$ and $\sigma_{n+1}$ and there is no nonzero piecewise linear function on the cones $\sigma_{0}, \sigma_{4 n+1}, \sigma_{6 n+1}$ and $\sigma_{1}, \ldots, \sigma_{n-1}, \sigma_{n}^{\prime}, \sigma_{n+2}, \ldots, \sigma_{2 n}$. Furthermore, if $n \geq 4$ we can remove cones $\sigma_{n-1}$ and $\sigma_{n+2}$ by removing them from the list of points defining $T$, respectively $P_{n-1}, Q_{n-1}$ and $P_{n+1}, Q_{n+1}$. This way we further decrease the value $a(X)$ by one or two. Thus we may obtain $a(X)=t$ for any $t \geq 5$.

\section{Further REMARKS}

It may be worth highlighting that the variety $X$ constructed in the proof of Theorem 3.1 shows that some of the intuitive, useful or classic theorems for smooth or $\mathbb{Q}$-factorial varieties fail to be true for normal varieties. We will give two examples:

Proposition 4.1 (Jelonek, 6, Proposition 2.2]). Let $X$ be a smooth complete nonprojective threefold and let $U \subset X$ be a maximal quasiprojective subset of $X$. Then the set $G:=X \backslash U$ has a pure dimension 1 .

For $X$ from Theorem 3.1 the complements of maximal quasiprojective open subsets consist of one or two points corresponding to the maximal cones removed from the fan.

Proposition 4.2 (Kleiman, [7, Chapter IV, §2, Theorem 2]). Assume $V$ is quasidivisorial. Then the ample cone $\stackrel{\circ}{P}(V)$ is the interior of the pseudoample cone $P(V)$.

For the variety from Theorem 3.1 the Néron-Severi space is 0-dimensional, so one might discard it as a special case. However if we take the product with a projective variety, then the pseudoample cone will have maximal (positive) dimension and the ample cone will remain empty. 
We still do not know whether the Chevalley conjecture as stated in Conjecture 1.1 is true. The variety from Theorem 3.1 shows that the classic Kleiman approach, or any other based on the value $\rho(X)$, must fail. However there are two conjectures which would imply Conjecture 1.1.

Conjecture 4.3 (Włodarczyk, [11, Conjecture 5.3]). Every normal variety $X$ satisfying $a(X)=\infty$ can be embedded in a normal toric variety satisfying $a(X)=\infty$.

The implication follows from the fact that every toric variety satisfying $a(X)=\infty$ is projective; projectivity follows from Theorems 3.3 and 3.4 and the fact that every toric variety is associated to a fan with finitely many maximal cones. Notice that Conjectures 1.1 and 4.3 are in fact equivalent, since the projective space is a toric variety and $a\left(\mathbb{P}^{n}\right)=\infty$.

Conjecture 4.4 (Białynicki-Birula, [1]). Any normal algebraic variety $X$ contains only finitely many subsets maximal in the family of all quasiprojective open subsets of $X$.

The implication is obvious since $a(X) \geq m q o s(X)$ implies projectivity.

\section{ACKNOWLEDGEMENT}

The author would like to thank Professor Jelonek for his advice and support.

\section{REFERENCES}

1. A. Białynicki-Birula, Finiteness of the number of maximal open subsets with good quotients, Transform. Groups, Vol. 3, No. 4, 1998, 301-319. MR1657520 (99m:14089)

2. M. Farnik, Z. Jelonek, A complete variety with infinitely many maximal quasiprojective open subsets, Demonstratio Math., 43 (2010), no. 2, 277-284. MR2668476 (2011j:14001)

3. W. Fulton, Introduction to toric varieties, Princeton Univ. Press, Princeton, New Jersey, 1993. MR 1234037 (94g:14028)

4. H. Hironaka, On the theory of birational blowing up, Thesis, Harvard, 1960.

5. Ch.D. Hacon, J. McKernan, Flips and flops, Proceedings of the International Congress of Mathematicians, 2010, 513-539. MR2827807

6. Z. Jelonek, On the projectivity of threefolds, Proc. Amer. Math. Soc., vol. 133, no. 9, 2005, 2539-2542. MR2146196 (2005m:14069)

7. S. Kleiman, Toward a numerical theory of ampleness, Annals of Math. 84, 1966, 293-344. MR0206009 (34:5834)

8. J. Kollár, Rational curves on algebraic varieties, Springer-Verlag, Berlin, Heidelberg, 1996. MR 1440180 (98c:14001)

9. G. Kempf, F. Knudsen, D. Mumford, B. Saint-Donat, Toroidal embeddings, Springer-Verlag, Berlin, Heidelberg, New York, Tokyo, 1978. MR0335518 (49 \#299)

10. I.R. Shafarevich, Basic Algebraic Geometry 2, Springer-Verlag, Berlin, Heidelberg, 1994. MR:1328834 (95m:14002)

11. J. Włodarczyk, Embeddings in toric varieties and prevarieties, J. Algebraic Geometry 2, 1993, 705-726. MR:1227474(94e:14070)

12. J. Włodarczyk, Maximal quasiprojective subsets and the Kleiman-Chevalley quasiprojectivity criterion, J. Math. Sci. Univ. Tokyo 6, 1999, 41-47. MR1683254 (2000i:14009)

Instytut Matematyczny, Polska Akademia Nauk, św. Tomasza 30, 31-027 Kraków, PolAnd

Current address: Instytut Matematyczny, Polska Akademia Nauk, Śniadeckich 8, 00-956 Warszawa, Poland

E-mail address: michal.farnik@gmail.com 